\title{
VALORACIÓN DE LA ADIPOSIDAD CORPORAL DE ESCOLARES EN AREQUIPA, PERÚ
}

\author{
Marco Antonio Cossio-Bolaños ${ }^{1,2, a}$, Carlos Pablos Abella ${ }^{3, b}$, Miguel Arruda ${ }^{1, a}$
}

\section{RESUMEN}

Objetivos. Evaluar la relación entre el porcentaje de grasa corporal, el índice de masa corporal y el perímetro del abdomen en una población de escolares que viven a moderada altitud. Materiales y métodos. Se realizó un estudio transversal para el cual fueron seleccionados de forma probabilística 394 niños y 405 niñas entre 6 y 11 años de edad. Todos los escolares seleccionados son de condición socioeconómica media y pertenecen al área urbana de Arequipa, Perú, localizada a moderada altitud (2320 m de altitud). Se evaluó el porcentaje de grasa, el índice de masa corporal y el perímetro del abdomen. La correlación fue evaluada con el coeficiente de correlación de Spearman. Resultados. Se observó correlación moderada entre el porcentaje de grasa y el perímetro del abdomen en niños $(0,59)$ y en niñas $(0,68)$. Se obtuvieron valores inferiores entre el porcentaje de grasa y el índice de masa corporal en niños $(0,56)$ y en niñas $(0,66)$. Se observó asociación entre las categorías del porcentaje de grasa (\%G) y el perímetro del abdomen $(p<0,05)$; sin embargo, no hubo asociación entre el \%G y el índice de masa corporal $(p>0,05)$. Conclusiones. El perímetro del abdomen tiene una correlación moderada con el porcentaje de grasa corporal y puede ser utilizado como un indicador de adiposidad de la región central del cuerpo en niños que viven en Arequipa (Perú) a moderada altitud.

Palabras clave: Adiposidad; Índice de masa corporal; Salud escolar; Altitud (fuente: DeCS BIREME).

\section{ASSESSMENT OF BODY ADIPOSITY IN SCHOOL STUDENTS IN AREQUIPA, PERU}

\begin{abstract}
Objectives. To assess the relation between the percentage of body fat, the Body Mass Index and the abdominal perimeter in a population of school students who live at a moderate altitude. Materials and methods. A cross-sectional study was conducted, for which 394 boys and 405 girls between 6 and 11 years of age were selected using a probabilistic method. All the school students chosen were middle class children who lived in the urban area of Arequipa, Peru, located at a moderate altitude (2320m above sea level). The percentage of fat, the Body Mass Index and the abdominal perimeter were assessed. Results. There was a moderate correlation between the percentage of fat and the abdominal perimeter in boys $(0.59)$ and girls (0.68). Lower figures were obtained between the percentage of fat and the Body Mass Index in boys (0.56) and girls (0.66). A connection among the categories of fat percentage $(\% \mathrm{~F})$ and the abdominal perimeter $(p<0.05)$ was observed; yet, there was no connection between the $\% \mathrm{~F}$ and the Body Mass Index $(\mathrm{p}>0.05)$. Conclusions. The abdominal perimeter has a moderate correlation with the percentage of body fat and can be used as an indicator of adiposity in the mid-section of the body of children who live in Arequipa (Peru) at a moderate altitude.
\end{abstract}

Key words: Adiposity; Body mass index; School health; Altitude (source: MeSH NLM).

\section{INTRODUCCIÓN}

La distribución de la grasa corporal ha sido asociada significativamente como elemento de predicción del factor de riesgo de enfermedades cardiovasculares en adultos de ambos sexos ${ }^{(1,2)}$. La relación existente entre ambas variables no solo está condicionada por la cantidad de tejido adiposo, sino también por el patrón de distribución de grasa en el cuerpo ${ }^{(3)}$. En este sentido, el exceso de grasa corporal localizada en la región abdominal (obesidad central) constituye un factor de riesgo más relevante que la propia obesidad ${ }^{(4)}$. De hecho, varios

\footnotetext{
Facultad de Educación Física, Universidad Estadual de Campinas. Sao Paulo, Brasil

Departamento de Ciencias de la Actividad Física, Universidad Católica del Maule, Chile.

Instituto de Ciencias de la Actividad Física y Deporte, Universidad Católica de Valencia, España.

a Licenciado en Educación Física doctor en Ciencias del Deporte; ${ }^{\text {b }}$ licenciado en Ciencias de la Actividad Física doctor en Ciencias de la Actividad Física y Deporte Recibido: 09-07-12 Aprobado: 14-11-12
}

Citar como: Cossio-Bolaños MA, Pablos Abella C, Arruda M. Valoración de la adiposidad corporal de escolares en Arequipa, Perú. Rev Peru Med Exp Salud Publica. 2012;29(4):477-82. 
estudios destacan un gran aumento del sobrepeso y obesidad en niños y adolescentes de diversas regiones del mundo ${ }^{(5-7)}$ inclusive en el Perú ${ }^{(8-10)}$.

Los efectos del sobrepeso y la obesidad abarcan alteraciones hormonales, metabólicas, ortopédicas y psicológicas (11,12); el incremento en el número de niños con problemas de sobrepeso y obesidad, conlleva a su vez al incremento en el riesgo de padecer tempranamente alguna enfermedad cardiometabólica ${ }^{(13)}$. En tal sentido, para la valoración de la adiposidad corporal en niños y adolescentes, la antropometría y las referencias internacionales de crecimiento son las herramientas más sencillas y de uso común para valorar el estado nutricional. De esa forma, la Organización Mundial de la Salud (OMS) ${ }^{(14)}$ señala a la antropometría como el método más útil para identificar personas con exceso de peso corporal, principalmente en estudios epidemiológicos, puesto que es económica y no invasiva, universalmente aplicable y con buena aceptación por la población. Dentro de los indicadores antropométricos que permitan una estimación razonable de distribución de la grasa normal se han propuesto varios parámetros, entre ellos la medición de los pliegues cutáneos y de los perímetros de diferentes segmentos corporales ${ }^{(15)}$, considerando algunos estudios como parámetros clásicos el índice de masa corporal (IMC), el perímetro de cintura y la relación perímetro de cintura/estatura ${ }^{(16,17)}$.

No obstante, la información relacionada a las metodologías de la valoración del sobrepeso y obesidad en niños y adolescentes del Perú aún es incipiente, sobre todo en niños que viven en regiones geográficas de altitud (aquellas ubicadas por encima de los $2000 \mathrm{~m}$ de altitud), ya que algunos estudios previos destacan que el IMC no sería aplicable a niños que viven a moderadas altitudes (entre los 2000 y 3000 m de altitud) (18), puesto que presentarían valores inferiores de estatura en relación a las referencias de la OMS y del Centro para el Control y la prevención de enfermedades (CDC). Por lo tanto, el estudio tiene objetivo evaluar la relación entre los parámetros de adiposidad, es decir entre el porcentaje de grasa corporal, el IMC y el perímetro del abdomen (PA) para la valoración de la adiposidad corporal de escolares que viven en Arequipa (Perú) a moderada altitud.

\section{MATERIALES Y MÉTODOS}

\section{TIPO DE ESTUDIO Y MUESTRA}

Se realizó un estudio transversal a partir de una base de datos secundaria, cuya muestra proviene del proyecto longitudinal denominado "Crecimiento físico y aptitud física de niños y adolescentes de zonas urbanas de
Arequipa, Perú", que tuvo como objetivo principal analizar la tendencia secular de variables de crecimiento físico y composición corporal. Se analizaron los datos de escolares comprendidos en un rango de edad entre 6,0 a 11,0 años. Los mismos fueron seleccionados de forma probabilística, para lo cual se diseñó un muestreo estratificado por afijación proporcional, tomando en cuenta una precisión del 5\%, con un nivel del confianza al 95\%; se consideró una prevalencia esperada del $50 \%$ para obtener el mayor tamaño de muestra. El tamaño final de la muestra fue de 799 sujetos; 394 niños y 405 niñas de un total de 5627 escolares (2790 niños y 2837 niñas).

Para la determinación del nivel socioeconómico se aplicó un instrumento que incluye siete preguntas con fiabilidad de 0,85 (alfa de Cronbach), los que permitieron identificar a los escolares de condición socioeconómica media por medio de una escala que va de 12 a 75 puntos; donde valores $\leq 35$ puntos indican condición baja, valores entre 35-59 puntos indican condición media y $\geq 60$ puntos especifican condición alta. El lugar de nacimiento se verificó mediante la partida de nacimiento otorgada por las instituciones educativas. Esta información permitió incluir a los niños que nacieron en Arequipa (Perú) a $2320 \mathrm{~m}$ de altitud. Los niños que nacieron en regiones geográficas de nivel del mar $(0,5 \%)$ fueron excluidos, siendo considerados en el estudio únicamente los niños que nacieron en la región y zonas aledañas ubicadas a $2320 \mathrm{~m}$ de altitud. Por lo tanto, el estudio incluye alumnos en edad escolar pertenecientes al sistema educativo peruano de educación básica regular (nivel primario).

\section{PROCEDIMIENTOS}

Para la evaluación de las variables antropométricas se adoptó el protocolo estandarizado de la International Working Group of Kineanthropometry descrita por Ross y Marfell-Jones ${ }^{(19)}$. Para el caso, todas las variables fueron evaluadas con la menor cantidad de ropa posible y sin calzado, los pliegues cutáneos tricipital y subescapular fueron medidas en el lado derecho del cuerpo.

La masa corporal $(\mathrm{kg})$ fue medida utilizando una balanza digital con una precisión de $200 \mathrm{~g}$ de marca Tanita $^{\circledR}$ con una escala de 0 a $150 \mathrm{~kg}$. La estatura $(\mathrm{cm})$ se evaluó ubicando el plano infraorbitariomeatal (Plano de Frankfur), se utilizó un estadiómetro de aluminio graduada en milímetros de marca Seca ${ }^{\circledR}$, presentando una escala de 0-2,50 m. Los pliegues cutáneos $(\mathrm{mm})$ de la región tricipital y subescapular fueron medidos de acuerdo a la línea de clivaje utilizando un compás de pliegues cutáneos Harpenden ${ }^{\circledR}$ que ejerce una presión constante de $10 \mathrm{~g} / \mathrm{mm}^{2}$. El PA (cm) se midió en la región abdominal en su mayor perímetro (a la altura del ombligo), 
se realizó a través de una cinta métrica de nylon (Seca $\left.{ }^{\circledR}\right)$, milimetrada y con una precisión de $(0,1 \mathrm{~cm})$. Para el diagnóstico del estado nutricional a partir del PA se utilizó los puntos de corte sugeridos por Frisancho ${ }^{(24)}$, donde se consideran como eutróficos del P15,1-P85,0; sobrepeso del P85,1-P95,0 y obeso del P95,1-P100.

El porcentaje de grasa $(\% \mathrm{G})$ se determinó por medio de la ecuación de regresión propuesta por Boileau, Lohman y Slaughter ${ }^{(20)}$, este indicador se utilizó como criterio principal para determinar la relación entre los demás indicadores de adiposidad. Para clasificar en categorías (bajo, normal, sobrepeso y obesidad) a partir del porcentaje de grasa se utilizó los puntos de corte propuestos por Heyward, Stolarczyk ${ }^{(21)}$ para niños en función del sexo.

Con el objetivo de relacionar el peso con la estatura se utilizó el índice de masa corporal. Se utilizó la fórmula propuesta por Quetelet [IMC=peso $(\mathrm{kg}) /$ estatura $\left.(\mathrm{m})^{2}\right]^{(22)}$. Los puntos de corte utilizados para la clasificación en categorías (bajo, normal, sobrepeso y obesidad) fueron adoptados según la propuesta de Cole et al. ${ }^{(23)}$ para niños de ambos sexos.

Se utilizó una doble medición cada diez sujetos en todas las variables (peso, estatura, PA, pliegues cutáneos tricipital y subescapular). Los valores del error técnico de la medida (ETM) oscilan entre 1-3\%, y el coeficiente de reproductibilidad fue de 0,97 a 0,99 . Todas las medidas fueron realizadas por un único evaluador con certificación ISAK nivel III.

\section{ANÁLISIS ESTADÍSTICO}

La distribución normal fue verificada a través de la prueba Shapiro Wilks, los resultados del estudio fueron analizados a través de la estadística descriptiva de media aritmética, desviación estándar, las diferencias entre sexos se verificó a través de la prueba t de Student para muestras independientes. Para correlacionar los indicadores de adiposidad se utilizó el coeficiente producto-momento de Pearson ( $r$ ), las diferencias significativas entre las categorías se determinaron a través de ANOVA de dos vías y la asociación entre el porcentaje de grasa con el IMC y el PA se verificó por medio de la prueba exacta de Fisher. En todos los casos se consideró un nivel de significancia de $(p<0,05)$. Se aplicó el método LMS para ajustar los datos asimétricos utilizando una distribución Box-Cox-normal, que sigue los datos empíricos exactamente.

\section{ASPECTOS ÉTICOS}

El estudio contó con la aprobación del Comité de Ética del Departamento Médico del Instituto del Deporte Universitario de la Universidad Nacional de San Agustín de Arequipa (Cod. 002-2009-IDUNSA-CIE); igualmente, los padres o tutores de los niños llenaron y firmaron la ficha de consentimiento informado autorizando las evaluaciones antropométricas.

\section{RESULTADOS}

Encontramos que los niños de ambos sexos muestran valores similares de IMC $\left(17,3 \pm 1,9 \mathrm{~kg} / \mathrm{m}^{2}\right.$ en niños y $17,3 \pm 2,2 \mathrm{~kg} / \mathrm{m}^{2}$ en niñas) y PA $(60,1 \pm 5,5 \mathrm{~cm}$ en niños y $60,2 \pm 8,9 \mathrm{~cm}$ en niñas); sin embargo, respecto al porcentaje de grasa corporal, las niñas $(25,0 \pm 4,4 \% \mathrm{G})$ muestran valores significativos más altos que los niños $(14,3 \pm 4,4 \% G)$, respectivamente $(p<0,01)$. Ello se aprecia con mayor claridad en la Tabla 1 en donde se describen las medidas antropométricas según edad y sexo, no hay diferencias de estas variables entre ambos

Tabla 1. Características antropométricas de los escolares estudiados.

\begin{tabular}{|c|c|c|c|c|c|c|}
\hline Edad (años) & N. ${ }^{\circ}$ & Masa corporal (kg) & Estatura (cm) & $\mathrm{PA}(\mathrm{cm})$ & $\% G$ & IMC \\
\hline \multicolumn{7}{|l|}{ Niños } \\
\hline $6,0-6,9$ & 56 & $21,8 \pm 2,2$ & $114,3 \pm 5,3^{*}$ & $54,5 \pm 2,8$ & $12,5 \pm 2,8^{*}$ & $16,7 \pm 1,4$ \\
\hline $7,0-7,9$ & 67 & $22,9 \pm 3,9$ & $117,5 \pm 5,1^{*}$ & $58,1 \pm 5,3$ & $12,7 \pm 4,0^{*}$ & $16,5 \pm 2,1$ \\
\hline $8,0-8,9$ & 68 & $25,8 \pm 3,6$ & $124,8 \pm 4,7$ & $58,8 \pm 4,0$ & $13,3 \pm 3,7^{*}$ & $16,6 \pm 1,8$ \\
\hline $9,0-9,9$ & 73 & $29,3 \pm 3,8$ & $127,6 \pm 4,4^{*}$ & $61,6 \pm 5,0$ & $14,3 \pm 4,6^{*}$ & $18,0 \pm 2,1$ \\
\hline $10,0-10,9$ & 59 & $33,2 \pm 4,1^{*}$ & $136,4 \pm 6,7^{*}$ & $62,9 \pm 5,1$ & $16,1 \pm 3,8^{*}$ & $17,8 \pm 1,4$ \\
\hline $11,0-11,9$ & 71 & $37,0 \pm 4,8$ & $142,6 \pm 5,4$ & $63,9 \pm 4,5$ & $16,9 \pm 4,3^{*}$ & $18,2 \pm 1,6$ \\
\hline Todos & 394 & $28,5 \pm 3,8$ & $127,2 \pm 5,4$ & $60,1 \pm 5,5$ & $14,3 \pm 4,4^{*}$ & $17,3 \pm 1,9$ \\
\hline \multicolumn{7}{|l|}{ Niñas } \\
\hline $6,0-6,9$ & 80 & $22,5 \pm 2,2$ & $118,8 \pm 5,1$ & $55,3 \pm 3,3$ & $17,4 \pm 3,3$ & $15,8 \pm 1,3$ \\
\hline $7,0-7,9$ & 61 & $24,6 \pm 3,5$ & $122,6 \pm 4,3$ & $58,1 \pm 5,4$ & $18,6 \pm 4,4$ & $16,3 \pm 1,5$ \\
\hline $8,0-8,9$ & 63 & $26,6 \pm 3,0$ & $126,4 \pm 6,1$ & $59,6 \pm 3,3$ & $20,7 \pm 2,8$ & $16,6 \pm 1,2$ \\
\hline $9,0-9,9$ & 58 & $30,4 \pm 4,6$ & $132,1 \pm 6,2$ & $60,7 \pm 4,2$ & $22,0 \pm 3,7$ & $17,4 \pm 2,1$ \\
\hline $10,0-10,9$ & 63 & $37,2 \pm 5,4$ & $140,2 \pm 5,7$ & $63,7 \pm 6,3$ & $21,3 \pm 4,6$ & $18,8 \pm 2,2$ \\
\hline $11,0-11,9$ & 76 & $38,9 \pm 5,9$ & $144,1 \pm 5,2$ & $64,4 \pm 5,9$ & $23,3 \pm 4,4$ & $18,7 \pm 2,3$ \\
\hline Todos & 401 & $30,1 \pm 4,1$ & $130,7 \pm 5,4$ & $60,2 \pm 8,9$ & $25,0 \pm 4,4$ & $17,3 \pm 2,2$ \\
\hline
\end{tabular}

PA: perímetro abdominal, \%G: porcentaje de grasa corporal, IMC: índice de masa corporal.

* Comparación entre sexos $(p<0,05)$ 

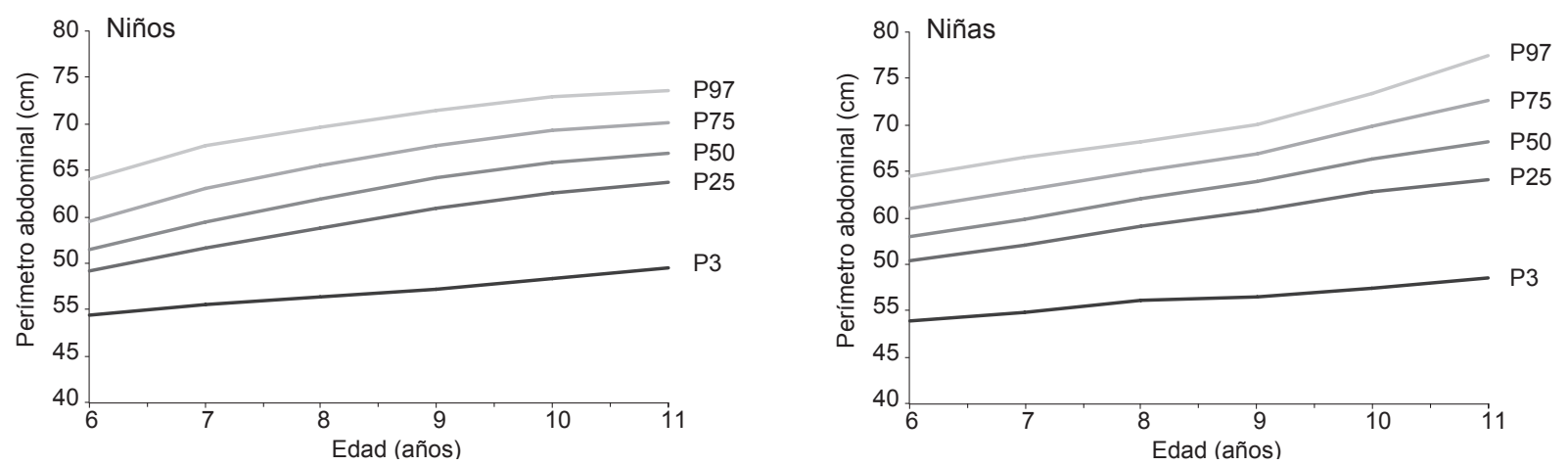

Figura 1. Distribución percentilar del perímetro abdominal $(\mathrm{cm})$ en niños que viven a moderada altitud.

sexos para una misma edad, excepto en el \%G, donde las niñas tienen valores mayores que los niños en todas las edades $(p<0,05)$.

En la Figura 1 se aprecian los cambios en los valores del PA expresados en percentiles. Estos valores permiten evaluar la adiposidad corporal de niños de ambos géneros de 6 a 11 años.

En niños, el PA tuvo una correlación moderada con el IMC $(0,62)$ y con el \%G $(0,59)$, siendo menor la correlación entre \%G e IMC $(0,56)$. En las niñas, las correlaciones se incrementaron, existiendo una alta correlación entre PA e IMC $(0,74)$, y moderada entre PA y $\% G(0,68)$ y entre $\%$ G e IMC $(0,66)$.

Las medias de las cuatro categorías determinadas a partir del PA se pueden distinguir en la Figura 2, en la cual se observan diferencias significativas entre las categorías para cada sexo $(p<0,05)$, sin embargo, cuando se comparó entre sexos, no se observó diferencias $(p>0,05)$.

El porcentaje de niños que se encuentran en cada categoría según la clasificación efectuada por el IMC y el PA en función del porcentaje de grasa corporal permitió determinar que el IMC no se asocia con el porcentaje de grasa $(p>0,05)$.

\section{DISCUSIÓN}

De acuerdo con los resultados obtenidos en el presente estudio, podemos destacar correlaciones moderadas entre los tres indicadores de adiposidad. El porcentaje de grasa en el estudio fue considerado como criterio de base para la valoración de la adiposidad corporal, a partir del cual verificamos que el PA muestra mayor correlación con el \%G en ambos sexos ( $r=0,59-0,68)$;
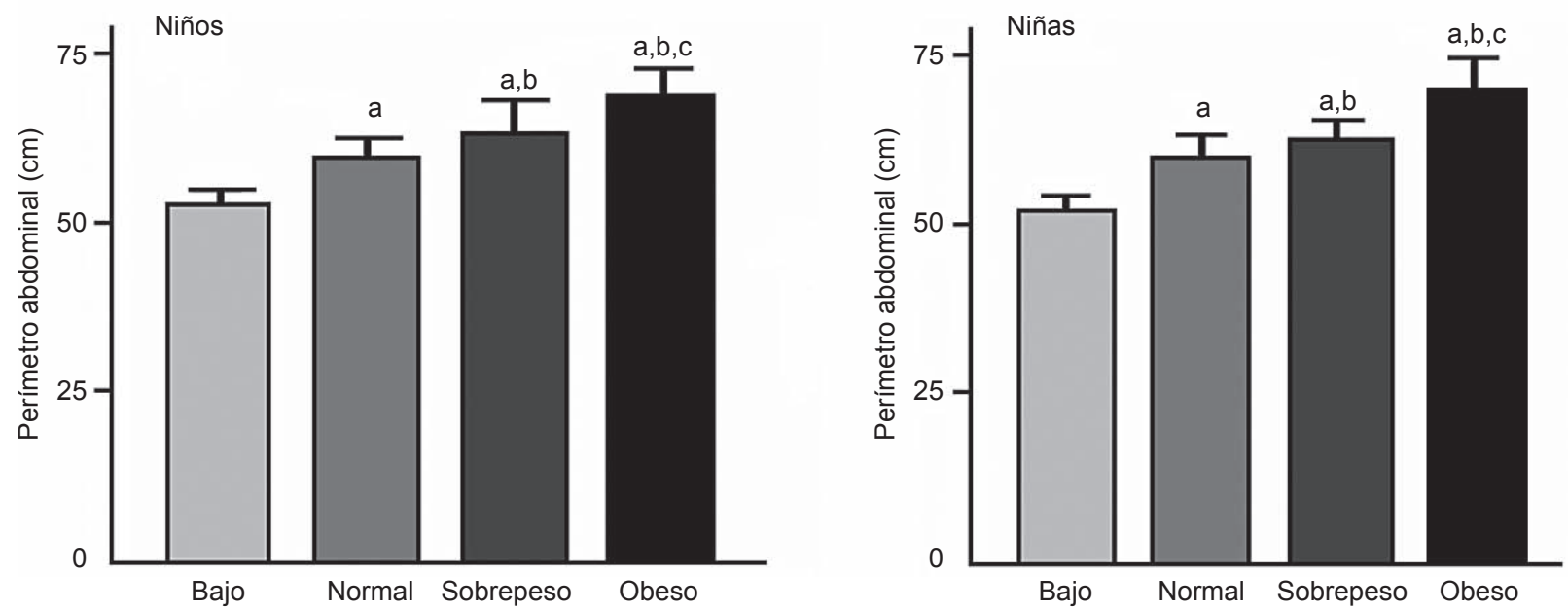

Figura 2. Perímetro abdominal según sexo y estado nutricional. a,b,c: $p<0,05$, a: con relación a la categoría de bajo, b: con relación a la categoría de normal, c: con relación a la categoría de sobrepeso. 
sin embargo, el IMC a pesar de ser usado y considerado por múltiples estudios como un indicador de adiposidad corporal en niños y adolescentes ${ }^{(25,26)}$, no mostró tal magnitud.

En general, el IMC se acepta como un método indirecto para estimar en niños, adolescentes y adultos la cantidad de tejido adiposo, a pesar de que la relación masa corporal/superficie no mide directamente este tejido ${ }^{(22)}$. De hecho, algunos estudios consideran que el uso del IMC de forma individual es limitado en la infancia y la adolescencia, sobre todo para identificar individuos con exceso de peso corporal (27), inclusive su uso en poblaciones de niños que viven en regiones de moderada altitud es cuestionable, ya que puede sobreestimar el exceso de grasa en individuos de baja estatura ${ }^{(18)}$. En este sentido, el PAes una posibilidad para valorar el exceso de grasa corporal en la región central del cuerpo, dado que algunos estudios consideran que el diagnóstico precoz ${ }^{(28,29)}$ podría contribuir en la prevención del desarrollo de algunas anomalías como síndrome metabólico, hiperinsulinemia, hipertensión, hiperlipidemia, diabetes mellitus tipo II y enfermedad cardiovascular arterioesclerótica. De hecho, los niños y adolescentes no escapan a esta tendencia, puesto que este incremento en la prevalencia está asociado a profundos cambios socioeconómicos, tecnológicos, biotecnológicos, poblacionales y familiares que han ocurrido en el mundo en las últimas dos o tres décadas y que afectan tanto a países desarrollados como a aquellos en vías de desarrollo, llevando a un balance energético positivo en una gran parte de la población ${ }^{(30)}$.

Por otro lado, son muy escasos los datos respecto a los pliegues cutáneos y otros indicadores de composición corporal en niños y adolescentes del Perú. Algunos estudios proporcionan información de sobrepeso y obesidad a partir del índice nutricional (9) e IMC $(8,10)$; sin embargo, estos resultados no permiten contrastar los nuestros, puesto que tales índices no distinguen el fraccionamiento de los compartimientos corporales ${ }^{(27)}$ y menos la distribución de la grasa corporal en la región central del cuerpo. Desde esa perspectiva, hay necesidad de crear y proponer criterios específicos para la valoración del crecimiento físico y del estado nutricional de la población peruana; sin embargo, el Perú es un país con diversas características geográficas y culturales, lo que limita la estandarización de cualquier tentativa, pero el incremento del exceso de peso infantil observado en el Perú durante los últimos años ${ }^{(31)}$ estimula a desarrollar nuevas metodologías para identificar el exceso de adiposidad corporal.
En nuestro estudio el IMC no mostró asociación con el $\%$ G, lo que limita su uso y aplicación para la valoración de la adiposidad corporal en este tipo de población. Por lo tanto, es una necesidad conocer el patrón de distribución de la grasa corporal en el abdomen en etapas tempranas de la vida ${ }^{(13)}$, puesto que su diagnóstico precoz posibilitaría ejercer políticas públicas en niños de regiones geográficas de moderadas y elevadas altitudes del Perú y, consecuentemente, aplicar programas de intervención con el propósito de preservar la salud de los escolares peruanos.

El estudio presenta algunas limitaciones, por ejemplo no fue posible controlar la presión arterial de los niños estudiados, puesto que hubiera permitido validar de alguna forma nuestros resultados, especialmente en el grupo de niños que fueron clasificados con sobrepeso y obesidad. Aunque en la actualidad se considera como estándar de oro la absorciometría fotónica por rayos-X $\operatorname{DEXA}^{(7)}$ para medir en forma directa la grasa corporal total, subcutánea y visceral. Este instrumento en nuestro medio es limitado por su alto costo y su escasa disponibilidad para la práctica cotidiana; sin embargo, el uso de las ecuaciones de regresión es una forma habitual para determinar el porcentaje de grasa corporal el cual muy bien podría ser usado como un criterio para relacionar con otras variables de adiposidad corporal. Sugerimos para futuros estudios ampliar el rango de edad y comparar con otras muestras de niños de regiones geográficas de moderadas y elevadas altitudes, así como utilizar otras variables antropométricas para la determinación de la adiposidad corporal en escolares del Perú.

En conclusión, el perímetro del abdomen muestra correlación moderada con el porcentaje de grasa corporal, y podría considerase su uso como un indicador de adiposidad de la región central del cuerpo en niños que viven a moderada altitud. Su valoración puede ser realizada a través de percentiles y puntuación- $Z$ en función de la edad y sexo.

Contribuciones de autoría: MACB participó en la concepción y diseño de trabajo, recolección y obtención de resultados, análisis e interpretación de datos y redacción del manuscrito. MA y CPA participaron en la concepción y diseño del trabajo, análisis estadístico y revisión crítica del manuscrito. Todos los autores aprobaron la versión final.

Fuentes de financiamiento: estudio financiado por La Coordinación de Perfeccionamiento del Personal de Nivel Superior CAPES, SP, Brasil.

Conflictos de interés: los autores declaran no tener conflictos de interés. 


\section{REFERENCIAS BIBLIOGRÁFICAS}

1. Martínez E, Devesa M, Bacallao J, Amador M. Índice subescapular/ tricipital: valores percentilares en niños y adolescentes cubanos. Arch Lat Nutr. 1993;43(3):199-203.

2. Ho SY, Lam TH, Janus ED; Hong Kong Cardiovascular Risk Factor Prevalence Study Steering Committee. Waist to stature ratio is more strongly associated with cardiovascular risk factors than other simple anthropometric indices. Ann Epidemiol. 2003;13(10):683-91.

3. Moreno LA, Fleta J, Sarría A, Rodríguez G, Gil C, Bueno M. Secular changes in body fat patterning in children and adolescents of Zaragoza (Spain), 19801995. Int J Obes Relat Metab Disord. 2001;25(11):1656-60.

4. Sinaiko A. Obesidade, resistência à insulina e síndrome metabólica. J Pediatr (Rio J). J Pediatr (Rio J). 2007;83(1):3-4.

5. Rolland-Cachera MF, Castetbon K, Arnault N, Bellisle F, Romano MC, Lehingue $\mathrm{Y}$, et al. Body mass index in 7-9-y-old French children: frequency of obesity, overweight and thinness. Int J Obesity. 2002;26(12):1610-6.

6. Núñez-Rivas HP, Monge-Rojas R, León $\mathrm{H}$, Roselló M. Prevalence of overweight and obesity among Costa Rican elementary school children. Rev Panam Salud Publica. 2003;13(1):24-32.

7. Lobstein T, Baur L, Uauy R; IASO International Obesity TaskForce. Obesity in children and young people: a crisis in public health. Obes Rev. 2004;5 Suppl 1:4-104.

8. Bustamante A, Seabra AF, Garganta RM, Maia JA. Efectos de la actividad física y del nivel socioeconómico en el sobrepeso y obesidad de escolares, Lima Este 2005. Rev Peru Med Exp Salud Publica. 2007;(24)2:121-8.

9. Cossio-Bolaños MA, EduardoHespanhol J, Merma MC, Arruda M. Desempeño de la condición física relacionada a la salud en función del índice nutricional en niños de moderada altitud. Pediatr Mex. 2011;13(3):96-102.

10. Rosado-Cipriano MM, Silvera-Robles VL, Calderón-Ticona JR. Prevalencia de sobrepeso y obesidad en niños escolares. Rev Soc Peru Med Interna. 2011;24(4):163-9.

11. Marrodán Serrano M, Mesa Santurino M, Alba Díaz JA, Ambrosio Soblechero
B, Barrio Caballero PA, Drak Hernández L, et al. Diagnosis de la obesidad: actualización de criterios y su validez clínica y poblacional. An Pediatr (Barc). 2006;65(1):5-14.

12. Ronque E, Serpeloni Cyrino E, Dórea V, Serassuelo Júnior $\mathrm{H}$, Gemente Galdi EH, Arruda M. Diagnóstico da aptidão física em escolares de alto nivel socioeconômico: avaliação referenciada por critérios de saúde. Rev Bras Med Esporte. 2007;13(2):71-6.

13. Schmidt MD, Dwyer T, Magnussen CG, Venn AJ. Predictive associations between alternative measures of childhood adiposity and adult cardiometabolic health. Int J Obes (Lond). 2011;35(1):38-45.

14. World Health Organization. Physical status: the use and interpretation of anthropometry. WHO Technical Report Series 854. Geneva: WHO; 1995.

15. Cuestas-Montañés E, Achával A, Garcés N, Larraya C. Circunferencia de la cintura, dislipidemia e hipertensión arterial en prepúberes de ambos sexos. An Pediatr (Barc). 2007;67(1):44-50.

16. Moreno LA, Pineda I, Rodríguez G, Fleta J, Sarría A, Bueno M. Waist circumference for the screening of the metabolic syndrome in children. Acta Pediatr. 2002;91(12):1307-12.

17. Hirschler V, Maccallini G, Calcagno M, Aranda C, Jadzinsky M. Waist circumference identifies primary school children with metabolic syndrome abnormalities. Diabetes Technol Ther. 2007;9(2):149-57.

18. Cossio-Bolaños MA, Maria TS; Campos RG; Pascoal EH; Eduardo-Hespanhol J; Arruda M. O uso das curvas de crescimento da Organização Mundial da Saúde em crianças e adolescentes que vivem em regiões de altitude moderada. Rev Paul Pediatr. 2012;30(3):314-320.

19. Ross WD y Marfell-Jones MJ. Kinanthropometry. In: J.D. MacDougall, H.A, Wenger, y H.J, Geen (Eds). Physiological testing of elite athlete. London: Human Kinetics; 1991. p. 223-308.

20. Boileau RA, Lohman TG, Slaughter $\mathrm{MH}$. Exercise and body composition in children and youth. Scan J Sports Sci. 1985;7:17-27.

21. Heyward VH, Stolarczyk LM. Appliedbody composition assement.
Champaign, Illinois: Human Kinetics; 1996.

22. Garrow JS, Webster J. Quetelet's index (W/H2) as a measure of fatness. Int J Obes. 1985;9(2):147-53.

23. Cole TJ, Bellizzi MC, Flegal M, Dietz WH. Establishing a standard definition for child overweight and obesity worldwide: international survey. BMJ. 2000;320(7244):1240-3.

24. Frisancho AR, Tracer DP. Standards of arm muscle by stature for assessment of nutritional status of children. Am J Phys Anthropol. 1987;73(4):459-65.

25. Dietz WH, Bellizzi MC. Introduction: the use of body mass index to assess obesity in children. Am J Clin Nutr. 1999;70(1):123S-5S.

26. Giugliano R, Carneiro EC. Fatores associados à obesidade em escolares. J Pediat. 2004;80(1):17-22.

27. Demerath EW, Schubert CM, Maynard LM, Sun SS, Chumlea WC, Pickoff A, et al. Do changes in body mass index percentile reflect changes in body composition in children? Data from the Fels Longitudinal Study. Pediatrics. 2006;117(3):e487-95.

28. Fagot-Campagna A, Pettitt DJ, Engelgau MM, Burrows NR, Geiss LS, Valdez R, et al. Type 2 diabetes among North Americanchildren and adolescents: an epidemiologic review and a public health perspective. J Pediatr: 2000;136(5):664-72.

29. Hirschler V, Aranda C, Calcagno ML, Maccalini G, Jadzinsky M. Can waist circumference identify children with the metabolic syndrome? Arch Pediatr Adolesc Med: 2005;159(8):740-4.

30. Consenso sobre factores de riesgo de enfermedad cardiovascular en pediatría. Obesidad. Arch Argent Pediatr. 2005;103(3):262-81.

31. Mispireta ML, Rosas AM, Velásquez JE, Lescano AG, Lanata CF. Transición Nutricional en el Perú, 1991 - 2005. Rev Peru Med Exp Salud Publica. 2007;24(2):129-35.

Correspondencia: Marco Antonio Cossio Bolaños. Dirección: Rua Érico Veríssimo 701, Cidade Universitária. Campinas/SP, 13083-851, São Paulo, Brasil

Teléfono: (5519) 35216750

Correo electrónico:mcossio1972@hotmail.com 